\title{
EDITORIAL
}

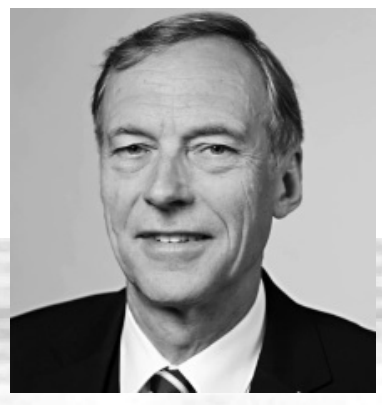

Arndt Bode

Hauptherausgeber

\section{Informatik und Evolution}

Ein starkes Plädoyer für mehr Forschung zum Thema der Software-Evolution halten Barbara Paech, Universität Heidelberg, Sven Apel, Universität Passau, Lars Grunske, Universität Stuttgart und Christian Prehofer, TU München und fortiss GmbH, die aus dem Schwerpunktprogramm 1593 der Deutschen Forschungsgemeinschaft mit dem Thema "Design for Future - Managed Software Evolution" berichten. Die Autoren wünschen sich eine Verstärkung von empirischer Informatikforschung zur Software-Evolution und zur Wirksamkeit der im Rahmen der vom Schwerpunktprogramm entwickelten Methoden und Werkzeuge. Sie plädieren für post-mortem-, in-vitro- und in-vivo-Studien für diese Evaluation.

Auch Frank J. Furrer, IT-Berater aus Stein am Rhein, befasst sich mit zukunftsfähigen Softwaresystemen unter Verwendung einer praktisch erprobten Evolutionsstrategie - die "Managed Evolution“. Derartig entwickelte Softwaresysteme sollen nicht nur aktuelle Anforderungen optimal erfüllen, sondern auch durch Evolution für längere Zeit erfolgreich, sicher und wirtschaftlich sein.

In der Student's Corner berichtet Sebastian Möller über sein Projekt im Medienlabor der Hochschule Osnabrück zu einem adaptiven Mehrbenutzer Informationssystem. Ziel ist es dabei, computergestützte Informationssysteme in öffentlichen Räumen so zu gestalten, dass auch gleichzeitig mehrere Benutzer individualisierte Informationen simultan erhalten können.

In der Rubrik „Zur Diskussion gestellt" stellt Heiko von Drachenfels, Hochschule Konstanz, die Frage: Um welche Probleme geht es eigentlich im Software-Engineering? Er befasst sich mit den Problemfeldern des Software Engineerings: Dekomposition, Abstraktion, Wiederverwendung, Redundanz, Abhängigkeiten, Formalisierung und äußere Rahmenbedingungen. Von Drachenfels präsentiert eine pragmatische Sicht, die auch dazu führen soll, dass Software Engineering nicht als Disziplinierung von Softwareentwicklern empfunden wird. Ich bin mir sicher, dass dieser Beitrag eine rege Diskussion auslösen wird.

Der Hauptbeitrag von Oliver Bendel, Fachhochschule Nordwestschweiz, behandelt Chancen und Risiken für Benutzer und Betroffene privater Drohnen. Diese ethische Fragestellung ist letztlich erst dadurch entstanden, dass die Informatik effiziente und effektive Programme zur Steuerung unbemannter Flugkörper entwickeln konnte. Bendel sieht viele Risiken privater Drohnen, aber auch einige Chancen. Er verweist darauf, dass zu diesen neuen Erscheinungsformen des täglichen Lebens ein gesellschaftlicher Diskurs stattfinden muss, dem dann aber auch vom Gesetzgeber ein passender Rahmen für die Nutzung privater Drohnen folgen muss. Die Gesellschaft für Informatik hat in ihrem Präsidiumsarbeitskreis „Datenschutz und IT-Sicherheit" bereits mehrfach zur Datenschutzproblematik Stellung genommen, wie der Autor zu Recht darstellt.

Ich wünsche erbauliches Lesevergnügen bei allen Hauptbeiträgen und den regelmäßigen Rubriken dieser Ausgabe des Informatik-Spektrums.

München, 24. März 2016

Arndt Bode

DOI 10.1007/s00287-016-0966-5 